\title{
Correlative Measurement of Color Centres in Nanodiamond Using Optical and Advanced Electron Microscopy and Spectroscopy
}

\author{
Shery Chang ${ }^{1}$, Haotian Wen ${ }^{2}$ and Christian Dwyer ${ }^{2}$ \\ ${ }^{1}$ University of New South Wales, Sydney, New South Wales, Australia, ${ }^{2}$ Arizona State University, \\ Tempe, Arizona, United States
}

Color centers such as nitrogen-vacancy (N-V) in diamond have been studied extensively for their interesting photoluminescence and spin properties. Negatively charged N-V centers (N-V-) can emit visible light that is readily detectable at the room temperature and its spin triplet states gives excellent spin properties allowing applications in quantum information technologies. Spectroscopy techniques such as electron paramagnetic resonance (EPR) have been used to identify different types of defect centers in diamond [1]. Locating the $\mathrm{N}-\mathrm{V}$ centers has becoming routine using optical fluorescence microscopy [2]. However it has been observed that the brightness and the spin properties of the color centers can vary significantly particularly for those close to the surface. These variations have been attributed to the changes in the host structure and the surface chemistry and structure.

In order to understand the origin of variations of optical and spin properties, we directly correlated the optical measurements with the atomic scale structural properties of nanodiamond materials by using highresolution imaging and electron energy loss spectroscopy in a (S)TEM, in combination with confocal fluorescence microscopy and spectroscopy on the same specimen area. We examined type $1 \mathrm{~b}$ diamond nanoparticles synthesized using high pressure high temperature (HPHT) processes. The surfaces of the particles were treated in order to enhance the optical properties. The particles were deposited on silicon nitride membrane TEM grids for both TEM and confocal fluorescence microscopy experiments.

High-resolution imaging was carried out using a monochromated, aberration-corrected TEM (Titan, FEI Company) operated at $80 \mathrm{kV}$. A monochromated, aberration corrected STEM (Nion UltraSTEM ${ }^{\mathrm{TM}}$ ) operated at $60 \mathrm{kV}$ was used for electron energy loss spectroscopy. Optical measurements was carried out using a custom-built scanning confocal fluorescence microscope with a high numerical aperture objective lens at room temperature. Both imaging and spectroscopy (steady-state and time-resolved fluorescence decay) have been obtained on this instrument.

We show that EELS can be used to identify the presence of $\mathrm{N}-\mathrm{V}$ centers in nanodiamond in individual nanodiamond particles. We have identify features in the C-kedge core loss spectra that is associated with the presence of point defects including the N-V centers [3]. Moreover we have also demonstrated that it is possible to measure the electronic transitions of the NV center in nanodiamond using high-energy resolution low-loss EELS [4].

In addition, by correlating the photoluminescence map directly with the HRTEM and EELS maps, we revealed the relationship of particle size, surface atomic and electronic structures with the brightness of individual particles [5]. This correlation gives us insight into how surface treatment might affect the optical properties of the color centers.

\section{References}

[1] S Felton et al, Phys. Rev. B 77(2008) p. 081201.

[2] C Bradac et al, Nature Nanotechnology 5(2010) p. 345. 
[3] S. L Y. Chang et al, Nanoscale (2016).

[4] C. Dwyer et al, (2020) manuscript pending.

[5] H.Wen et al, (2020) manuscript pending. 